\title{
Gateable Suppression of Spin Relaxation in Semiconductors
}

\author{
J. S. Sandhu, ${ }^{1}$ A.P. Heberle, ${ }^{2, *}$ J. J. Baumberg, ${ }^{3, \dagger}$ and J. R. A. Cleaver ${ }^{1}$ \\ ${ }^{1}$ Microelectronics Research Centre, Cavendish Laboratory, University of Cambridge, Cambridge CB3 OHE, United Kingdom \\ ${ }^{2}$ Hitachi Cambridge Laboratory, Cavendish Laboratory, Madingley Road, Cambridge CB3 OHE, United Kingdom \\ ${ }^{3}$ Department of Physics \& Astronomy, University of Southampton, Southampton, SO17 1BJ, United Kingdom
}

(Received 19 September 2000)

\begin{abstract}
The decay of spin memory in a 2D electron gas is found to be suppressed close to the metal-insulator transition. By dynamically probing the device using ultrafast spectroscopy, relaxation of optically excited electron spin is directly measured as a function of the carrier density. Motional narrowing favors spin preservation in the maximally scattered but nonlocalized electronic states. This implies that the spinrelaxation rate can be both tuned in situ and specifically engineered in appropriate device geometries.
\end{abstract}

DOI: $10.1103 /$ PhysRevLett.86.2150

Emerging technologies for generating and manipulating electronic spins are heralded as the precursor to a new generation of spin-functional devices [1]. One of the key requirements is to find a way to control electron spin in semiconductors which can be independently gated. Much work has been devoted to generating spins in semiconductors either optically [2], by injection through magnetic semiconductors [3-5], or within ferromagnetic semiconductors [6], while the transport of spin has also been shown to be feasible $[7,8]$. However, the relaxation of carrier spin is ascribed to several competing mechanisms and particularly at low temperatures has been found to vary widely between different samples [9-13]. One reason for this is that spin relaxation can be particularly sample dependent since it is strongly influenced by impurities and defects. It is therefore desirable that the same sample be used within a study. However, circumstances may prevent this, as in investigations of the dependence of spin relaxation on background doping level, where different wafers must be used [14]. In addition, such structures have no means for tuning the spin relaxation in a prescribed way.

In this Letter, we show an advantageous method which avoids the use of multiple samples to vary the number of carriers in a semiconductor by applying a reverse bias to a semitransparent Schottky contact situated above a charge transport layer. The effect of the gate is to transform the quasi-2D electron gas (2DEG) from a high-density metallic state to a low-density insulating state. At low temperatures it is possible to use this technique to reduce the conductivity by several orders of magnitude. This large change in conductivity is, however, accompanied by a relatively small change in electron density, typically less than an order of magnitude. It has been realized that the remote ionized donors that provide the electrons for the 2DEG play an important role in this drop in conductivity [15-17]. Spatial variations in the density of these donors result in random fluctuations in the potential in the plane of the 2DEG. In the conducting regime, the $2 \mathrm{DEG}$ acts to screen these fluctuations. However, as the density is reduced the screening becomes less effective and the fluctuations grow. This manifests itself as electron localization and corresponds to
PACS numbers: 78.47.+p, 42.40.My, 71.30. $+\mathrm{h}, 73.21 .-\mathrm{b}$

a large decrease in conductivity. In this Letter, the spinrelaxation dynamics of photoexcited carriers are investigated as we pass through this metal-insulator $(M-I)$ phase transition from a conducting metallic state to an insulating state. We show that the presence of the random scattering potential suppresses the spin relaxation, due to the orientational averaging of direction-dependent spin torques.

The heterostructure used for this study was grown by molecular beam epitaxy with the layer structure: $n$-doped substrate, followed by $2 \mu \mathrm{m}$ of $\mathrm{Al}_{0.3} \mathrm{Ga}_{0.7} \mathrm{As}, 25 \mathrm{~nm} \mathrm{GaAs}$ quantum well, $40 \mathrm{~nm} \mathrm{Al}_{0.3} \mathrm{Ga}_{0.7} \mathrm{As}$ spacer layer, $40 \mathrm{~nm}$ $\mathrm{Al}_{0.3} \mathrm{Ga}_{0.7} \mathrm{As}$ Si-doped $10^{18} \mathrm{~cm}^{-3}$ and finally a $10 \mathrm{~nm}$ GaAs cap layer. Standard lithographic methods were used to define a mesa forming a $400 \mu \mathrm{m} \times 400 \mu \mathrm{m}$ long cross, with $100 \mu \mathrm{m}$ wide bars, on which Ohmic contacts were made to the electron gas using $75 \mathrm{~nm}$ of $\mathrm{Au} / \mathrm{Ge} / \mathrm{Ni}$ covered by $150 \mathrm{~nm}$ of Au alloyed at $450{ }^{\circ} \mathrm{C}$ for $10 \mathrm{~s}$. A $100 \mu \mathrm{m} \times 100 \mu \mathrm{m}$ semitransparent Schottky contact was formed on top of the center of the mesa by evaporating $15 \mathrm{~nm}$ of Au at $5 \times 10^{-7}$ mbar [Fig. 1(a), inset]. The carrier density in the channel beneath the gate is determined [18] by measuring the small-signal capacitance of the device as a function of gate voltage [Fig. 1(a), $T=10 \mathrm{~K}$ ], giving a carrier concentration, $n=1.4 \times 10^{11} \mathrm{~cm}^{-2}$ with the gate bias, $V_{g}=0 \mathrm{~V}$. Below a bias of $-0.4 \mathrm{~V}$ the carrier density begins to reduce, and once $-1.0 \mathrm{~V}$ is applied the channel is pinched off. The points in Fig. 1(a) show the result of a self-consistent solution of the PoissonSchrödinger equations, which matches well with the experimentally determined values.

Photoluminescence (PL) spectra are taken at $10 \mathrm{~K}$ with $100 \mu \mathrm{W}$ HeNe laser excitation under various bias conditions [Fig. 1(b)]. As the gate bias is decreased, the peak at the $h h$ exciton energy is enhanced above the background PL (which has been subtracted). This feature is also present in photoconductivity (PC) spectra and is associated with the formation of excitons. Comparing Figs. 1(a) and 1(b), it can be seen that the onset of exciton formation begins at a relatively high carrier concentration $(5 \times$ $10^{10} \mathrm{~cm}^{-2}$ ). Because excitons are screened out at such high carrier concentrations, we surmise that the electron 


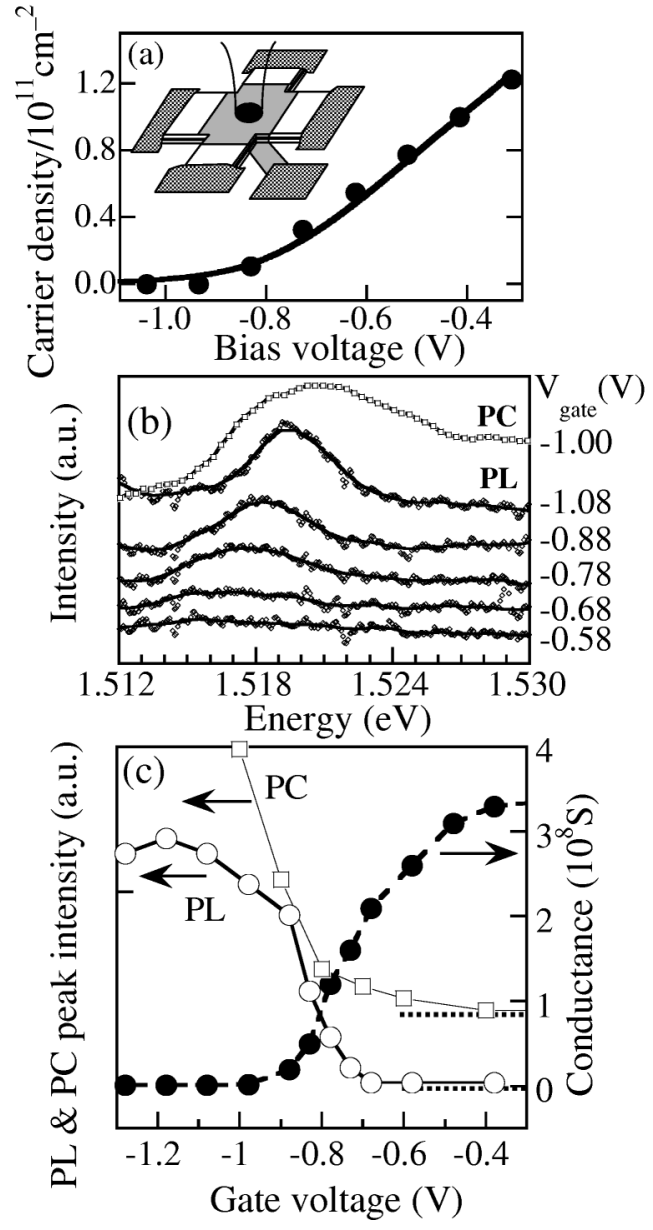

FIG. 1. (a) Carrier concentration vs gate bias derived from capacitance-voltage measurements (line). Points are derived by self-consistently solving the Poisson-Schrödinger equations. Inset: sample mesa. (b) Bias-dependent PL and PC for localization $(-1 \mathrm{~V})$ at the heavy-hole exciton energy from the gated mesa region with $100 \mu \mathrm{W}$ of HeNe excitation. The collection area is $(50 \mu \mathrm{m})^{2}$. A voltage-independent PL background has been subtracted from the spectra. (c) Bias-dependent conductivity, and peak amplitude of both PL and PC (zero marked with dotted lines), as a function of gate voltage. The drop in conductivity and rise of optical emission are signatures of the $M-I$ transition.

distribution of the 2DEG at this point is inhomogeneous. To quantify this, the conductance of the sample as a function of gate bias is plotted together with the variation of the PL and PC peaks in Fig. 1(c). The rise of the exciton peak is strongly correlated with the decrease of the conductance. We can therefore conclude in agreement with previous work [19] that the exciton line appears at the onset of the $M-I$ transition. As the 2DEG goes through the $M-I$ transition it separates into multiply disconnected regions, which have either metallic or insulating properties. The excitons are formed in the regions that are void of electrons. Such a model was predicted by Efros [15] and experimentally verified by Finkelstein [19]. In this model the remote ionized donors, randomly distributed in the doped region, cause electrostatic fluctuations of the potential in the plane of the 2DEG. As a negative bias is applied, the Fermi level becomes lower than the bottom of the conduction band in some regions, and it is in these areas that excitons can be created. Where screening becomes ineffective at the $M-I$ transition [15], the average potential fluctuations are $\left\langle U^{2}(r)\right\rangle \simeq 2 \pi e^{4} \varepsilon^{-2} N_{d} \ln (l / 2 s) \sim(19 \mathrm{meV})^{2}$, where $\varepsilon$ is the dielectric constant, $N_{d+}$ is the sheet donor concentration $\left(4 \times 10^{12} \mathrm{~cm}^{-2}\right), l$ is the long length cutoff and is assigned as the distance between the channel and the gate $(90 \mathrm{~nm})$, and $s$ is the spacer width $(40 \mathrm{~nm})$. The mean potential fluctuation is much larger than the exciton binding energy of $9 \mathrm{meV}$ thus forcing localization of the carriers and contributing to the observed PL linewidth. This is further supported by the threefold reduction in PC linewidth above the $M-I$ transition.

The method used to probe the spin polarization is alloptical time-resolved Faraday rotation (TRFR) $[20,21]$ depicted in Fig. 2(a). A population of spin-polarized carriers is photoexcited using a circularly polarized pump pulse [2] and the subsequent spin decay is monitored by tracking the rotation of the reflected linearly polarized probe pulse as a function of time delay [Fig. 2(b)]. Signal to noise is improved by using an optical bridge, which measures small deviations from the unrotated-probe, balanced condition. Both the pump and probe pulses are derived from a mode-locked Ti:sapphire laser with pulse duration $100 \mathrm{fs}$, the time delay is varied using a conventional delay line, and the probe pulse is attenuated to less than $20 \%$ of the pump-pulse power $\left(I_{0}=100 \mu \mathrm{W}\right)$. Both beams are focused to $50-\mu \mathrm{m}$-diameter spots centered on the mesa. The excitation energy is centered at $1.536 \mathrm{eV}$, which is $16 \mathrm{meV}$ above the lowest heavy-hole to electron transition and ensures that the carriers are always excited above the Fermi level. Changing the excess energy does not significantly change the spin-relaxation lifetime, which is extracted by fitting an exponentially decaying function with

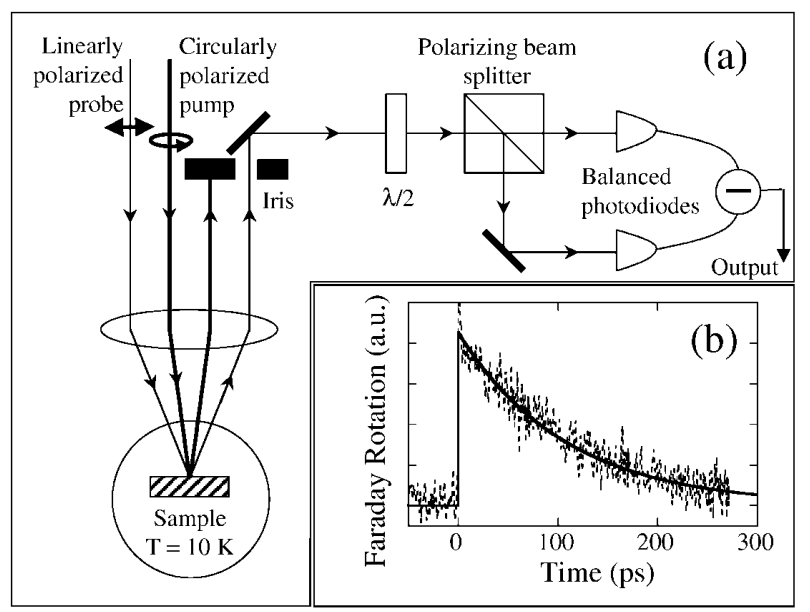

FIG. 2. (a) Experimental layout for TRFR measurements. The sample is cooled to $10 \mathrm{~K}$, and the submilliradian rotation of the reflected probe pulse is monitored as a function of time delay, $\tau$. (b) $\operatorname{TRFR}(\tau)$ signal, which tracks the electron spin relaxation, and exponential fit. 
a single decay time to the data (solid line). Carrier recombination does not contribute to our signal, as also indicated by the absence of biexponential relaxation rates and timeresolved reflection data on this type of sample. Changing the direction of pump-beam helicity and observing a change of signal sign verified the authenticity of the signal. Any long-lived nuclear spin polarizations are suppressed by the $50 \mathrm{kHz}$ modulation of the pump-beam helicity used for lock-in detection.

As the gate bias is varied, the electron spin-relaxation rate is modified, as shown clearly in Fig. 3(a) where the total carrier concentration in the channel, $N$, is the sum of the background electron concentration $(n)$ and the photoexcited carrier concentration $\left(N_{e x}=10^{9} \mathrm{~cm}^{-2}\right)$. A small lateral bias of $1 \mathrm{mV}$ is applied to simultaneously monitor the channel conductance. Figure 3(a) reveals the key result that the spin-relaxation rate is a nonmonotonic function of carrier concentration. As the carrier concentration is initially reduced, the spin-relaxation rate decreases until a critical concentration of $n_{c} \sim 5 \times 10^{10} \mathrm{~cm}^{-2}$ is reached, beyond which further reduction of the carrier concentration results in an increase in the spin-relaxation rate. This abrupt transition region corresponds to the $M-I$ phase transition region that was determined earlier. In the metallic phase, the carriers are unbound and, since hole spin scattering and recombination is fast at these excess energies [10], the electron spin-scattering rate dominates the TRFR signal. Below the phase transition, carrier screening disappears, so that electrons are trapped at sites opposite ionized donors in the remote donor layer [15]. The holes are repelled from the donors and lose their initial spin orientation quickly due to strong spin-orbit mixing, so the electron spin relaxation is again probed in this regime. Hence spin relaxation is clearly seen to be strongly influenced by the degree of scattering and localization of the confined electronic wave functions. Simple control using a gate allows access to a number of spin-transport regimes within a single heterostructure and points the way to spatially localized manipulation of spin memory.

In order to understand the spin decay in detail we consider the mechanisms involved in spin scattering. There are three main processes that contribute to spin relaxation in semiconductors, due to Elliot and Yafet (EY) [22], to D'yakonov and Perel' (DP) [23], and to Bir, Aronov, and Pikus (BAP) [24]. The EY mechanism arises out of spin-orbit scattering during collisions with phonons or impurities. For this mechanism the spin-relaxation rate increases with increasing momentum-scattering rate. This process is dependent on carrier concentration only through the average kinetic energy of the electrons. In a similar way, however, Coulomb scattering between electrons can lead to a substantial spin relaxation and has been shown to depend explicitly on the carrier concentration [25]; $\frac{1}{\tau_{s}} \propto \Gamma_{e-e} \propto N(k T)^{1 / 2}$. The DP mechanism results from the lack of inversion symmetry in zinc blende structures, which together with spin-orbit coupling gives a
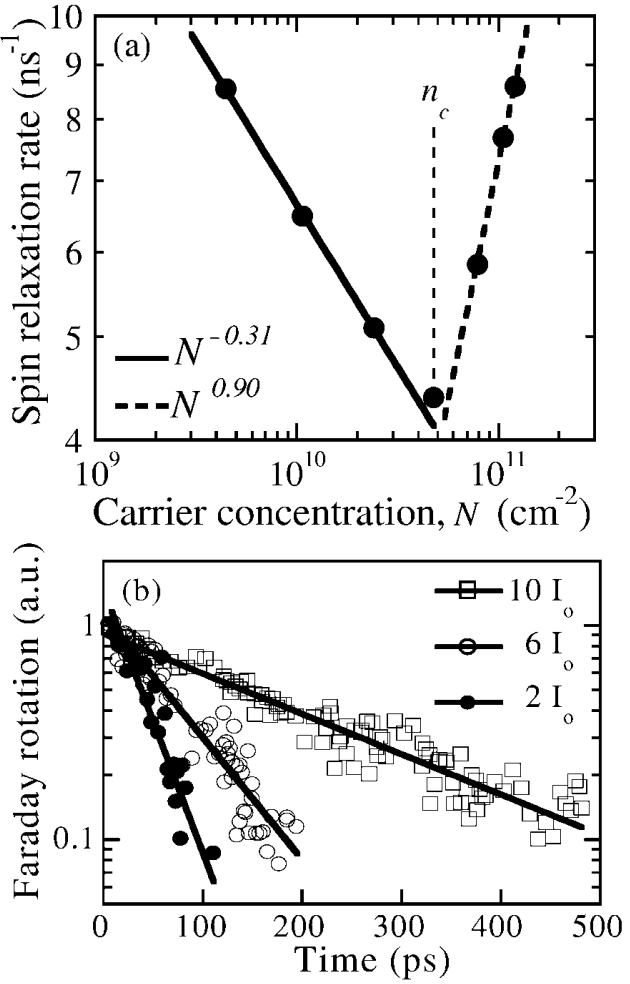

FIG. 3. (a) Nonmonotonic spin relaxation rate vs carrier concentration in the channel, fit by $N^{0.9}, N>n_{c}$ and $N^{-0.31}$, $N<n_{c}$. (b) High intensity power dependence of the spin relaxation at $N \sim 1 \times 10^{11} \mathrm{~cm}^{-2}$. The maximum pump power is an order of magnitude more than that used in (a) and generates a maximum carrier density at least an order of magnitude below the 2DEG electron density. The probe power was held constant.

$k$-dependent splitting between the spin components of the conduction band for $k \neq 0$. This splitting can be viewed as a pseudomagnetic field that causes a precession of electron spins. Momentum-scattering events cause fluctuations in the field seen by each electron that reduce the spin depolarization. In this mechanism the spin-relaxation rate is now inversely related to the momentum-scattering rate. Finally, the BAP mechanism arises from interaction of the electron spins with a population of unpolarized holes via the exchange interaction. This process is not strongly $k$ dependent $[26,27]$. Since the exchange interaction couples the electrons to a valence-band-mediated spin-relaxation channel, the decay rate is proportional to the wave function overlap between electron and hole.

We interpret the spin-relaxation data as follows. In the absence of an external bias with the carrier concentration high, scattering events are reduced by the efficient screening of the 2DEG. The suppressed momentum-scattering rate allows the DP mechanism to dominate. As the carrier density is reduced, screening becomes less effective thus increasing the electronic scattering from the random potential of ionized donors, which averages out the spin torques from the anisotropic lattice [2]. Increasing the optically injected carrier density, however, has a very different effect, due to the excess carrier energy and the additional 
holes which together increase carrier scattering rather than the screening. Thus at higher optical powers, increased scattering improves the "motional narrowing," extending the carrier lifetime again [Fig. 3(b)]. Further experiments are needed to confirm that the effects from hole momentum scattering dominate BAP spin exchange. The conductivity near the pinchoff bias is predicted [15] to follow $\sigma \sim\left(\frac{n}{n_{c}}-1\right)^{1.2}$, which leads to a spin-relaxation rate roughly proportional to $N$ for $N>n_{c}$ as observed experimentally. As expected we find a dramatic change as soon as the carriers are localized, which prevents this momentum scattering from enhancing the electron spin memory. Below the $M-I$ transition, the electrons become progressively more localized, sampling higher $k$ states, which leads to more mixing between the spin states (as shown theoretically for excitons [28]). In addition, the reduced screening of the ionized donors leads to a potential which fluctuates on a smaller length scale, thus allowing the hole to approach closer to the electron and increase the BAP spin scattering as the carrier density drops further. The scale length of the potential which controls this electron-hole overlap is given by the screening length of the 2DEG [15], $r_{s} \propto n^{-1 / 6}$, predicting a spin-scattering rate $\frac{1}{\tau_{s}} \propto r_{s}^{2} \propto n^{-1 / 3}$, also consistent with the data for $N<n_{c}$. A similar spin decay dependence is implied when the spin mixing $\propto k^{2}$ for the increasingly localized electrons. Changes in the electron-hole overlap in the growth direction, to which BAP is sensitive, are found to be minimal within this bias regime. A theory encompassing all spin-scattering mechanisms for the whole range of extended and localized states of electrons (and excitons [13]) is necessary to explain our results.

Several simple predictions are, however, possible. First, we predict that samples which enhance the momentumscattering rate without localizing the carriers can enhance the spin lifetime, in agreement also with experiments on bulk samples of different donor concentration [14]. Thus spin is preserved by aiming for a disorder potential which has a small barrier height and a high spatial frequency. The optimum spin memory is found at the $M-I$ transition, $n_{c} \sim 0.1 \sqrt{N_{d+}} / s$, so that the maximum spin lifetime is $\tau_{s} \propto N_{d+} / n_{c} \propto s \sqrt{N_{d+}}$, favoring the introduction of a high density of nonmagnetic remote scatterers. More generally, phase transitions are suggested to be ideal situations for protecting particular quantum degrees of freedom (such as spin) since the critical enhancement in fluctuations can lead to strong suppression of anisotropic perturbations through motional narrowing.

In conclusion, we have investigated the electron spin relaxation through the metal-insulator phase transition in an $n$-doped $\mathrm{GaAs} / \mathrm{AlGaAs}$ quantum well heterostructure. Appropriate device design allows time-resolved Faraday rotation measurements to track spin-relaxation rates directly while applying a variable reverse bias to the gate electrode. Enhanced spin memory is found at the metalinsulator phase transition, while carrier screening is detrimental to spin preservation. Further work at higher temperatures will examine how phonon scattering modifies this picture. The interplay between different spinrelaxation mechanisms within a single device points to the need to integrate a range of theoretical models.

The authors thank D. G. Hasko, J. Allam, B.W. Alphenaar, M. D. R. Thomas, and R. T. Harley for discussions and suggestions. J.S.S. acknowledges UK EPSRC support for this work.

*Email address: heberle@phy.cam.ac.uk †Email address: j.j.baumberg@soton.ac.uk

[1] G. A. Prinz, Phys. Today 48, No. 4, 58 (1995).

[2] Optical Orientation, edited by F. Meir and B.P. Zachachrenya (North-Holland, Amsterdam, 1984).

[3] M. Oestreich et al., Appl. Phys. Lett. 74, 1251 (1999).

[4] Y. Ohno et al., Nature (London) 402, 790 (1999).

[5] R. Fiederling et al., Nature (London) 402, 787 (1999).

[6] H. Ohno, Science 281, 951 (1998).

[7] D. Hagele, M. Oestreich, W. W. Rühle, N. Nestle, and K. Eberl, Appl. Phys. Lett. 73, 1580 (1998).

[8] M.E. Flatte and J.M. Byers, Phys. Rev. Lett. 84, 4220 (2000).

[9] For a review on spin relaxation in low-dimensional systems: L. Viña, J. Phys. Condensed Matter 11, 5929 (1999), and references therein.

[10] B. Baylac et al., Solid State Commun. 93, 57 (1995).

[11] S. Bar-Ad and I. Bar-Joseph, Phys. Rev. Lett. 68, 349 (1992).

[12] Ph. Roussignol et al., Phys. Rev. B 46, 7292 (1992).

[13] M. J. Snelling et al., J. Lumin. 45, 208 (1990).

[14] J. M. Kikkawa and D. D. Awschalom, Phys. Rev. Lett. 80, 4313 (1998).

[15] A. L. Efros, Solid State Commun. 70, 253 (1989).

[16] J. A. Nixon and J. H. Davies, Phys. Rev. B 41, 7929 (1990).

[17] A. Gold, Phys. Rev. B 44, 8818 (1991).

[18] J. Hillibrand and R. D. Gold, RCA Rev. 21, 245 (1960); Physics and Technology of Heterojunction Devices, edited by D. V. Morgan and R. H. Williams (Peter Peregrinus, London, 1991), pp. 125-134.

[19] G. Finkelstein, H. Shtrikman, and I. Bar-Joseph, Phys. Rev. Lett. 74, 976 (1995).

[20] J. J. Baumberg, D. D. Awschalom, and N. Samarth, J. Appl. Phys. 75, 6199 (1994).

[21] S. A. Crooker, D. D. Awschalom, J. J. Baumberg, F. Flack, and N. Samarth, Phys. Rev. B 56, 7574 (1997).

[22] R. J. Elliot, Phys. Rev. 96, 266 (1954); Y. Yafet, in Solid State Physics, edited by F. Seitz and D. Turnbull (Academic, New York, 1963), Vol. 14, p. 1.

[23] M. I. D'yakanov and V. I. Perel', Sov. Phys. JETP 33, 1053 (1971).

[24] G. L. Bir, A. G. Aronov, and G. E. Pikus, Sov. Phys. JETP 42, 705 (1976).

[25] P. Boguslawski, Solid State Commun. 33, 389 (1980).

[26] Ph. Roussignol et al., Surf. Sci. 267, 360 (1992).

[27] L. Munoz, E. Perez, L. Vina, and K. Ploog, Phys. Rev. B 51, 4247 (1995).

[28] M.Z. Maialle, Phys. Rev. B 61, 10877 (2000). 